\title{
Absolute neutrino mass as the missing link to the dark sector
}

\author{
Thede de Boer $\odot,{ }^{1}$ Michael Klasen $\odot,{ }^{1}$ Caroline Rodenbeck $\odot,{ }^{2}$ and Sybrand Zeinstra ${ }^{1}$ \\ ${ }^{1}$ Institut für Theoretische Physik, Westfälische Wilhelms-Universität Münster, \\ Wilhelm-Klemm-Straße 9, 48149 Münster, Germany \\ ${ }^{2}$ Institut für Kernphysik, Westfälische Wilhelms-Universität Münster, \\ Wilhelm-Klemm-Straße 9, 48149 Münster, Germany
}

(Received 13 July 2020; accepted 8 September 2020; published 24 September 2020)

\begin{abstract}
With the KATRIN experiment, the determination of the absolute neutrino mass scale down to cosmologically favored values has come into reach. We show that this measurement provides the missing link between the Standard Model and the dark sector in scotogenic models, where the suppression of the neutrino masses is economically explained by their only indirect coupling to the Higgs field. We determine the linear relation between the electron neutrino mass and the scalar coupling $\lambda_{5}$ associated with the dark neutral scalar mass splitting to be $\lambda_{5}=3.1 \times 10^{-9} m_{\nu_{e}} / \mathrm{eV}$. This relation then induces correlations among the dark matter (DM) and new scalar masses and their Yukawa couplings. Together, KATRIN and future lepton flavor violation experiments can then probe the fermion DM parameter space, irrespective of the neutrino mass hierarchy and $C P$ phase.
\end{abstract}

DOI: 10.1103/PhysRevD.102.051702

\section{INTRODUCTION}

The identification of cold dark matter (DM) — a mysterious particle that according to most cosmological models is five times more abundant in the Universe than ordinary matter-is one of the most urgent challenges in modern physics. Neutrinos as only weakly interacting massive particles in the Standard Model (SM) have the right characteristics of a DM candidate, but are neither cold nor can they, due to their tiny masses, contribute more than a small fraction (between 0.5 and $1.6 \%$ ) to the measured total DM relic density [1,2]. Nevertheless the idea that neutrinos and DM might be related is intriguing and has led to an enormous theoretical activity on so-called radiative seesaw models, where the suppression of the SM neutrino masses is due to their only indirect interaction (via DM) with the SM Higgs field [3-5].

While the fact that at least two of the three neutrino flavors are massive has been deduced about 20 years ago from atmospheric [6] and solar [7,8] neutrino oscillations, their absolute masses are still unknown. The KATRIN experiment has recently improved the upper limit on the electron (anti)neutrino mass to $1.1 \mathrm{eV}$ [9] and ultimately aims at a sensitivity of $0.2 \mathrm{eV}$ [10]. This value would rival the cosmological constraint on the sum of the SM neutrino masses of $\sum_{i} m_{\nu_{i}}<0.12 \mathrm{eV}$, assuming the $\Lambda \mathrm{CDM}$ model

Published by the American Physical Society under the terms of the Creative Commons Attribution 4.0 International license. Further distribution of this work must maintain attribution to the author(s) and the published article's title, journal citation, and DOI. Funded by SCOAP. and normal hierarchy $(\mathrm{NH})$, the minimal value allowed by oscillation experiments being $0.06 \mathrm{eV}[11,12]$. An inverted hierarchy (IH) is still a possibility, although the longbaseline experiments $\mathrm{T} 2 \mathrm{~K}$ and $\mathrm{NO} \nu \mathrm{A}$ and further evidence from reactor and atmospheric neutrinos favor $\mathrm{NH}$. For the $C P$ phase, $\mathrm{T} 2 \mathrm{~K}$ and to a lesser degree also $\mathrm{NO} \nu \mathrm{A}$ data seem to favor $3 \pi / 2(\pi / 2)$ for $\mathrm{NH}(\mathrm{IH})$ [13].

In radiative seesaw models, the SM neutrinos $\nu$, even under a discrete $Z_{2}$ symmetry, interact with the (also $Z_{2}$-even) SM Higgs field $\phi$ and obtain their masses via a dark ( $Z_{2}$-odd) sector, which contains only a small number of new multiplets (typically up to four new scalar/fermion singlets, doublets or triplets under $\left.\mathrm{SU}(2)_{L}\right)$ [4]. In Ma's famous scotogenic model (see Fig. 1), only one additional scalar doublet $\eta$ and (for three massive SM neutrinos) three generations of fermion singlets $N_{i}$ (sterile neutrinos with $i=1,2,3)$ are required [3]. The parameter space is therefore much smaller than, e.g., the one of supersymmetry and can be better constrained with neutrino oscillation data via the Casas-Ibarra method [14], limits on

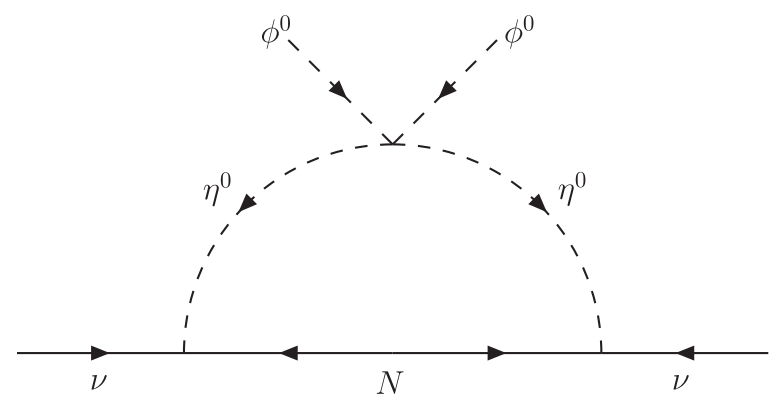

FIG. 1. Neutrino mass generation in scotogenic models. 
lepton flavor violation (LFV) [15], and measurements of the DM relic density [16]. Nevertheless, these previous analyses found that the dark scalar/fermion masses as well as their scalar and Yukawa couplings could still vary over several orders of magnitude.

In this paper, we demonstrate that a determination of the absolute electron neutrino mass, which has now come into reach, will provide additional stringent constraints on the dark sector of the scotogenic model in a way that is almost independent of the neutrino hierarchy and $C P$ phase. In particular, we determine the linear relation between the absolute electron neutrino mass and the scalar coupling associated with the mass splitting of the dark neutral scalars. This linear dependence induces correlations among the other parameters of the model, i.e., the DM and scalar masses and their Yukawa couplings, which we can also quantify. Together, current neutrino mass and future LFV experiments can then probe almost the entire fermion DM parameter space.

\section{THE SCOTOGENIC MODEL}

In the original scotogenic model, the SM is enlarged with a dark sector containing only two new types of fields, a complex Higgs doublet $\eta$ and three generations of fermion singlets $N_{i}$ [3]. In the Lagrangian of this model

$$
\mathcal{L}_{N}=-\frac{m_{N_{i}}}{2} N_{i} N_{i}+y_{i \alpha}\left(\eta^{\dagger} L_{\alpha}\right) N_{i}+\text { H.c. }-V,
$$

we define fermions in terms of Weyl spinors and denote the three generations of left-handed SM lepton doublets with $L_{\alpha}(\alpha=1,2,3)$. The fermion singlet with the smallest mass $m_{N_{i}}$ is assumed to be the DM candidate. SM neutrinos $\nu$ couple to the SM Higgs field $\phi$ and obtain their mass only at one loop (see Fig. 1) via the $3 \times 3$ matrices of Yukawa couplings $y_{i \alpha}$ and the scalar potential

$$
\begin{aligned}
V= & m_{\phi}^{2} \phi^{\dagger} \phi+m_{\eta}^{2} \eta^{\dagger} \eta+\frac{\lambda_{1}}{2}\left(\phi^{\dagger} \phi\right)^{2}+\frac{\lambda_{2}}{2}\left(\eta^{\dagger} \eta\right)^{2}+\lambda_{3}\left(\phi^{\dagger} \phi\right) \\
& \times\left(\eta^{\dagger} \eta\right)+\lambda_{4}\left(\phi^{\dagger} \eta\right)\left(\eta^{\dagger} \phi\right)+\frac{\lambda_{5}}{2}\left[\left(\phi^{\dagger} \eta\right)^{2}+\left(\eta^{\dagger} \phi\right)^{2}\right] .
\end{aligned}
$$

The parameters $m_{\phi}$ and $\lambda_{1}$ are fixed by the known SM Higgs vacuum expectation value (VEV) $\left\langle\phi^{0}\right\rangle=246 \mathrm{GeV} /$ $\sqrt{2}$ [1] and the LHC measurement of the (squared) SM Higgs boson mass $m_{h}^{2}=2 \lambda_{1}\left\langle\phi^{0}\right\rangle^{2}=-2 m_{\phi}^{2}=(125 \mathrm{GeV})^{2}$ [17]. To ensure that the scalar potential is bounded from below and the vacuum is stable, we must have

$$
\begin{gathered}
\lambda_{1}>0, \quad \lambda_{2}>0, \quad \lambda_{3}>-\sqrt{\lambda_{1} \lambda_{2}}, \\
\lambda_{3}+\lambda_{4}-\left|\lambda_{5}\right|>-\sqrt{\lambda_{1} \lambda_{2}},
\end{gathered}
$$

while perturbativity imposes $\left|\lambda_{2,3,4,5}\right|<4 \pi$. The inert doublet $\eta$ does not acquire a VEV, so that $\lambda_{2}$ induces only self-interactions and decouples from the phenomenology.
We set $\lambda_{2}=0.5$ without loss of generality. The masses of the charged scalar component $\eta^{+}$and the real and imaginary parts of the neutral component $\eta^{0}=\left(\eta_{R}+i \eta_{I}\right) / \sqrt{2}$ are then

$$
\begin{aligned}
m_{\eta^{+}}^{2} & =m_{\eta}^{2}+\lambda_{3}\left\langle\phi^{0}\right\rangle^{2}, \\
m_{R}^{2} & =m_{\eta}^{2}+\left(\lambda_{3}+\lambda_{4}+\lambda_{5}\right)\left\langle\phi^{0}\right\rangle^{2}, \\
m_{I}^{2} & =m_{\eta}^{2}+\left(\lambda_{3}+\lambda_{4}-\lambda_{5}\right)\left\langle\phi^{0}\right\rangle^{2} .
\end{aligned}
$$

Note that it is natural for $\lambda_{5}$ and the mass difference $m_{R}^{2}-m_{I}^{2}=2 \lambda_{5}\left\langle\phi^{0}\right\rangle^{2}$ to be small, since if $\lambda_{5}$ was exactly zero, it would induce a conserved lepton number and massless neutrinos [18]. Following previous work [16], we scan over the range $10^{-12}<\left|\lambda_{5}\right|<10^{-8}$. For vanishing $\lambda_{3}$, the LEP limit on charged particles [19] implies a lower limit on the scalar mass range $m_{\eta} \in[0.1 ; 10] \mathrm{TeV}$, which we also employ for the sterile neutrino masses $m_{N_{i}}$. As $m_{\eta}^{2}$ dominates over $\left\langle\phi_{0}\right\rangle^{2}$ in much of the parameter space, the scalar couplings $\lambda_{3}$ and $\lambda_{4}$ will play a subdominant role, and $\eta^{+}$will be close in mass to both $\eta_{R}$ and $\eta_{I}$.

\section{EXPERIMENTAL CONSTRAINTS}

The SM neutrino mass matrix $\left(m_{\nu}\right)_{\alpha \beta}=\left(y^{T} \Lambda y\right)_{\alpha \beta}$ can be written in a compact form using the Yukawa matrix $y$ and the diagonal mass matrix $\Lambda$ with eigenvalues

$$
\Lambda_{i}=\frac{m_{N_{i}}}{32 \pi^{2}}\left[\frac{m_{R}^{2}}{m_{R}^{2}-m_{N_{i}}^{2}} \log \left(\frac{m_{R}^{2}}{m_{N_{i}}^{2}}\right)-(R \rightarrow I)\right] .
$$

It is diagonalized by the Pontecorvo-Maki-NakagawaSakata (PMNS) matrix $U$,

$$
U^{T} m_{\nu} U=\hat{m}_{\nu} \equiv \operatorname{diag}\left(m_{1}, m_{2}, m_{3}\right) .
$$

This implies that, for a given set of masses in $\Lambda_{i}$, the Yukawa couplings

$$
y=\sqrt{\Lambda^{-1}} R \sqrt{\hat{m}_{\nu}} U^{\dagger}
$$

can be constrained, up to an orthogonal matrix $R$ depending on three arbitrary rotation angles $\theta_{i} \in[0 ; 2 \pi]$ [14], on top of the perturbativity bound $\left|y_{i \alpha}\right|^{2}<4 \pi$ and vacuum stability requirements [20], by the measured neutrino mass differences and mixing angles, which we apply at $3 \sigma$ [13]. When $\lambda_{5} \ll 1$ and $m_{R}^{2} \approx m_{I}^{2}$, the SM neutrino mass matrix simplifies to

$$
\begin{aligned}
\left(m_{\nu}\right)_{\alpha \beta} \approx & 2 \lambda_{5}\left\langle\phi^{0}\right\rangle^{2} \sum_{i=1}^{3} \frac{y_{i \alpha} y_{i \beta} m_{N_{i}}}{32 \pi^{2}\left(m_{R, I}^{2}-m_{N_{i}}^{2}\right)} \\
& \times\left[1+\frac{m_{N_{i}}^{2}}{m_{R, I}^{2}-m_{N_{i}}^{2}} \log \left(\frac{m_{N_{i}}^{2}}{m_{R, I}^{2}}\right)\right],
\end{aligned}
$$

i.e., it is not only bilinear in $y$, but also linear in $\lambda_{5}$. 
We then impose current (and study the constraining power of future) upper bounds on the most important LFV processes

$$
\begin{aligned}
\mathrm{BR}(\mu \rightarrow e \gamma) & <4.2 \times 10^{-13}[21]\left(2 \times 10^{-15}[22]\right), \\
\mathrm{BR}(\mu \rightarrow 3 e) & <1.0 \times 10^{-12}[23]\left(10^{-16}[24]\right), \\
\mathrm{CR}(\mu-e, \mathrm{Ti}) & <4.3 \times 10^{-12}[25]\left(10^{-18}[26]\right) .
\end{aligned}
$$

The branching ratios (BRs) and conversion rates (CRs) depend on the charged scalar and sterile neutrino masses and their Yukawa couplings through dipole/nondipole form factors and box diagrams [15] and are calculated with SPheno 4.0.3 [27].

We also restrict the DM relic density with micrOMEGAs 5.0.8 [28] to the central value $\Omega h^{2}=0.12$ measured by Planck [12], allowing for a theoretical uncertainty of 0.02 $[29,30]$. In the standard freeze-out scenario, the relic density results from DM annihilation processes in the early Universe, here of the lightest sterile neutrinos into leptonic final states via charged and neutral scalars in the $t$-channel. Coannihilation processes, which may occur in fine-tuned scenarios with nearly mass-degenerate scalars and fermions [31], are required to contribute less than $1 \%$. Direct DM detection is theoretically possible at one loop, but is currently beyond the experimental reach [32].

\section{NUMERICAL RESULTS}

For electron neutrino masses of $1.1 \mathrm{eV}$ to $0.2 \mathrm{eV}$ as currently explored by KATRIN $[9,10]$, i.e., larger than the minimal $\sum_{i} m_{\nu_{i}}>0.06 \mathrm{eV}$, but approaching the cosmological upper limit of $0.12 \mathrm{eV}[11,12]$, the mass differences, PMNS matrix $U$ and rotation angles $\theta_{i}$ play a subdominant role, and the eigenvalues of the Yukawas matrices $y_{\alpha}$ take

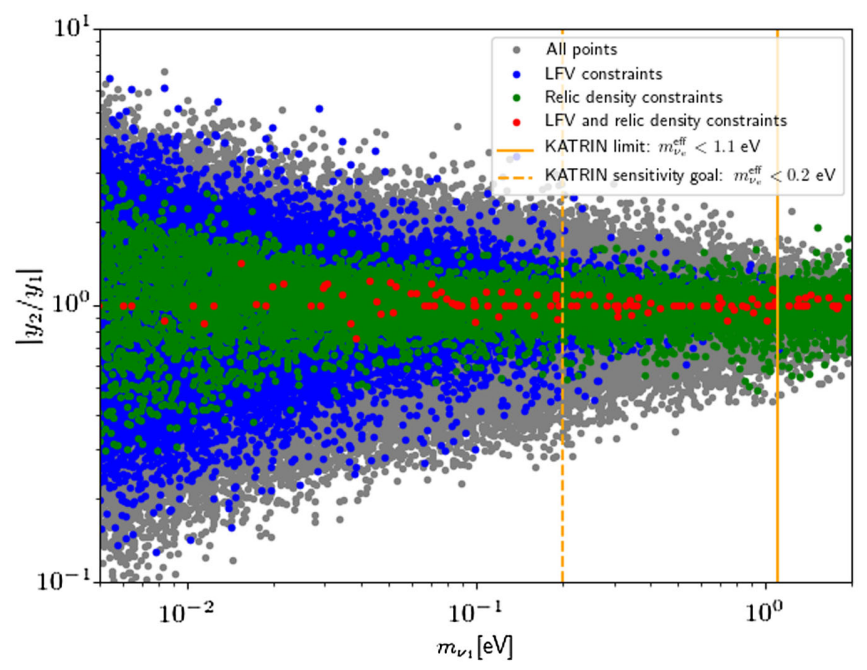

FIG. 2. Ratio of Yukawa couplings as a function of the lightest neutrino mass $m_{\nu_{1}}$ with mass difference/mixing (grey), LFV (blue), relic density (green) and all constraints (red points). similar values. This is demonstrated in Fig. 2 (grey points), where the ratio $\left|y_{2} / y_{1}\right|$ varies over its full range at low $m_{\nu_{1}}$, but only by about a factor of two at large $m_{\nu_{1}}$. In addition, the LFV processes $l_{\alpha} \rightarrow l_{\beta} \gamma$ and $l_{\alpha} \rightarrow 3 l_{\beta}$ impose upper limits on both $y_{\alpha}$ and $y_{\beta}$, limiting their ratio further (blue). Conversely, to obtain the correct DM relic density, the Yukawas must not be too small (green), so that the combination of all constraints leads indeed to $\left|y_{2} / y_{1}\right| \sim 1$ (red points). We have verified that this result is independent of the neutrino mass hierarchy and holds also for the ratios $\left|y_{3} / y_{1}\right|$ and $\left|y_{3} / y_{2}\right|$.

The linear dependence of the neutrino mass matrix $\left(m_{\nu}\right)_{\alpha \beta}$ on the dark sector-Higgs boson coupling $\left|\lambda_{5}\right|$ in Eq. (8) can then be made explicit by studying the dependence of $\left|\lambda_{5}\right|$ on the lightest eigenvalue $m_{\nu_{1}}$. It emerges in Fig. 3 after imposing LFV (blue), relic density (green) and all (red points) constraints and can be fitted at $90 \%$ C.L. to

$$
\left|\lambda_{5}\right|= \begin{cases}(3.08 \pm 0.05) \times 10^{-9} m_{\nu_{1}} / \mathrm{eV} & (\mathrm{NH}) \\ (3.11 \pm 0.06) \times 10^{-9} m_{\nu_{1}} / \mathrm{eV} & (\mathrm{IH})\end{cases}
$$

the sign being arbitrary, while below $m_{\nu_{1}}=0.052 \mathrm{eV}$ the heaviest neutrino mass dominates and

$$
\left|\lambda_{5}\right|= \begin{cases}(1.6 \pm 0.7) \times 10^{-10} & (\mathrm{NH}) \\ (1.7 \pm 1.5) \times 10^{-10} & (\mathrm{IH})\end{cases}
$$

becomes independent of $m_{\nu_{1}}$. The dark sector-Higgs boson coupling $\lambda_{5}$ can therefore be predicted, once the absolute neutrino mass scale is known.

Furthermore, with $m_{\nu_{1}} /\left|\lambda_{5}\right|$ fixed, the Yukawas in Eq. (8) become correlated with the DM and scalar masses. Since



FIG. 3. The dark sector-Higgs boson coupling $\left|\lambda_{5}\right|$ as a function of lightest neutrino mass $m_{\nu_{1}}$ with LFV (blue), relic density (green) and all constraints (red points). 


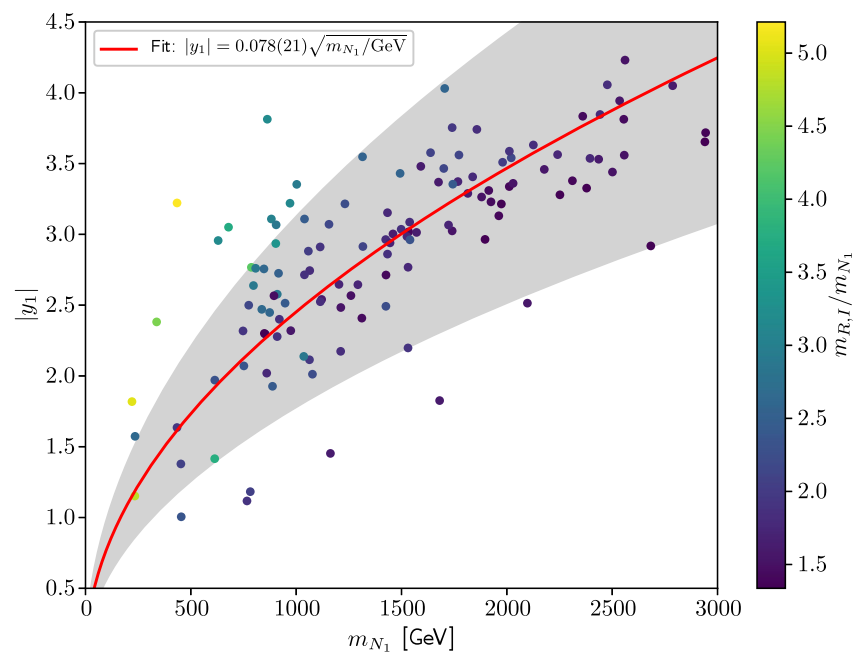

FIG. 4. Yukawa coupling of the lightest neutrino as a function of the DM mass. The ratio of the neutral scalar over the DM mass is given on the temperature scale.

the ratio of the latter is in addition constrained by the relic density $\left(m_{R, I} / m_{N_{1}} \sim 1.5\right)$, the leading term in Eq. (8) becomes proportional to $\left|y_{1}\right|^{2} / m_{N_{1}}$, which allows us to fit this dependence in Fig. 4 at $90 \%$ C.L. as

$$
\left|y_{1}\right|=\left\{\begin{array}{ll}
(0.078 \pm 0.021) \sqrt{m_{N_{1}} / \mathrm{GeV}} & (\mathrm{NH}) \\
(0.081 \pm 0.012) \sqrt{m_{N_{1}} / \mathrm{GeV}} & (\mathrm{IH})
\end{array},\right.
$$

the other fermions $N_{2,3}$ being significantly heavier. This implies, that if the DM mass is known, we can predict its Yukawa coupling to the SM leptons.

Our findings imply that the fermion DM parameter space of the scotogenic model can be almost completely tested with LFV experiments and a measurement or limit on the direct neutrino mass, as can be seen from Fig. 5. The current limit on $\operatorname{BR}(\mu \rightarrow e \gamma)$ (blue) [21] imposes stronger bounds than the one for $\operatorname{BR}(\mu \rightarrow 3 e)$ (red) [23], but this is expected to change soon $[22,24]$. Independently of the neutrino mass hierarchy, the models that survive even these future tight constraints can be probed in an orthogonal way by new limits or measurements of the absolute neutrino

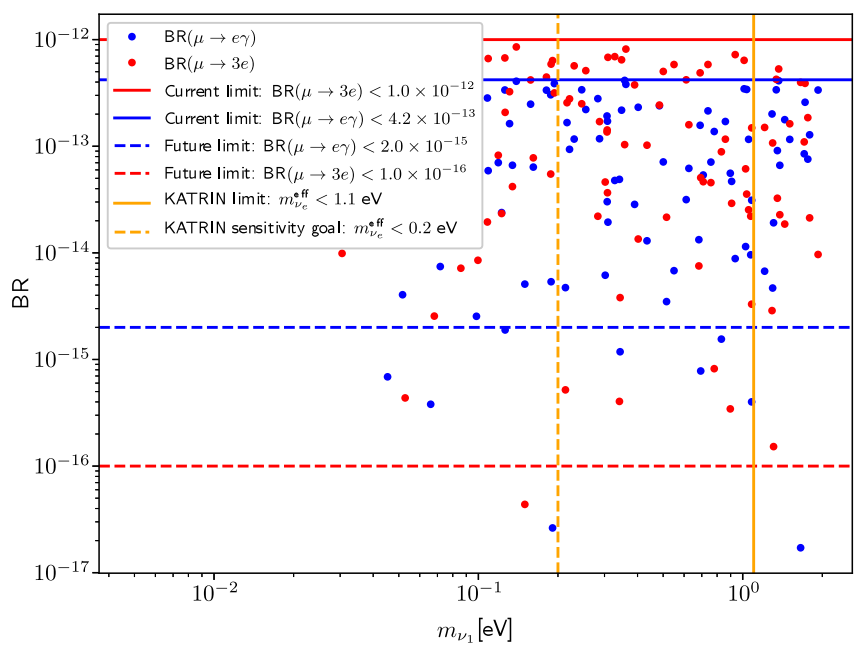

FIG. 5. Branching ratios of viable scotogenic models for the LFV processes $\mu \rightarrow e \gamma$ (blue) and $\mu \rightarrow 3 e$ (red points), their current (full) [21,23] and future (dashed) [22,24] experimental limits, and the current [9] and future [10] KATRIN limits (yellow lines) on the (effective) electron neutrino mass.

mass, if they reach indeed the region of cosmologically favored values $[9,10]$.

In Table I, we show the input parameters for a typical benchmark point at the KATRIN sensitivity limit (all masses in $\mathrm{GeV}$ ). The corresponding physical parameters, i.e., neutrino masses and mixings, relic density and branching ratios/conversion rate for this $\mathrm{NH}$ benchmark point (all masses in $\mathrm{eV}$, angles in ${ }^{\circ}$ ) are shown in Table II. We have checked that our results, in particular for the relic density, depend only weakly on $C P$-violating phases, so that they have been neglected. As one can see, this point fulfils all current constraints and is in the sensitivity range of forthcoming upgrades of the LFV experiments.

\section{SUMMARY AND OUTLOOK}

As we have demonstrated in this paper, the parameter space of fermion DM in Ma's scotogenic model is now severely constrained. In particular, an electron neutrino mass measurement would allow us to directly predict the dark sector-Higgs boson coupling $\lambda_{5}$ and to test the

TABLE I. Input parameters for a typical benchmark point at the KATRIN sensitivity limit (all masses in GeV).

\begin{tabular}{|c|c|c|c|c|c|c|c|c|c|c|c|c|c|c|c|c|c|}
\hline$m_{N_{1}}$ & $m_{N_{2}}$ & $m_{N_{3}}$ & $y_{11}$ & $y_{12}$ & $y_{13}$ & $y_{21}$ & $y_{22}$ & $y_{23}$ & $y_{31}$ & $y_{32}$ & $y_{33}$ & $m_{\eta}$ & $\lambda_{1}$ & $\lambda_{2}$ & $\lambda_{3}$ & $\lambda_{4}$ & $\lambda_{5}$ \\
\hline 1926 & 3773 & 3607 & -3.055 & -2.471 & 0.183 & 1.748 & -2.003 & 2.038 & -1.180 & 1.690 & 2.673 & 3371 & 0.26 & 0.5 & 7.576 & 6.060 & $6.2 \times 10^{-10}$ \\
\hline
\end{tabular}

TABLE II. Neutrino masses and mixings, relic density and branching ratios/conversion rate for our NH benchmark point (all masses in $\mathrm{eV}$, angles in ${ }^{\circ}$ ).

\begin{tabular}{cccccccccc}
\hline \hline$m_{\nu_{1}}$ & $\Delta m_{21}^{2}$ & $\Delta m_{31}^{2}$ & $\theta_{12}$ & $\theta_{23}$ & $\theta_{13}$ & $\Omega h^{2}$ & $\mathrm{BR}(\mu \rightarrow e \gamma)$ & $\mathrm{BR}(\mu \rightarrow 3 e)$ & $\mathrm{CR}(\mu-e, \mathrm{Ti})$ \\
\hline \hline 0.2 & $7.231 \times 10^{-5}$ & $2.444 \times 10^{-3}$ & 32.03 & 44.28 & 8.50 & 0.120 & $2.990 \times 10^{-13}$ & $8.321 \times 10^{-13}$ & $3.180 \times 10^{-13}$ \\
\hline \hline
\end{tabular}


complete parameter space of the model in an orthogonal way to LFV, while a DM mass measurement would result in a prediction for its Yukawa coupling to the SM leptons. This is due to the strong mutual constraints inherent in the one-loop diagram (Fig. 1) for neutrino mass generation, which is topologically similar to a penguin diagram mediating LFV and (when cut on the internal fermion line) to DM annihilation. The correlations are absent for scalar $\mathrm{DM}$, i.e., when the diagram is cut on the internal scalar lines, since the scalar doublets can annihilate into weak gauge bosons. The case of scalar-fermion coannihilation has been studied elsewhere [31].

In particular, we extended previous findings [16] in three different directions, showing that the eigenvalues of the Yukawa couplings are actually of very similar size with ratios close to unity; exposing the linear relation of $\lambda_{5}$ to the absolute electron neutrino mass as currently measured by KATRIN, while previously only neutrino mass differences were taken into account; and demonstrating the approximate square-root dependence of the Yukawa couplings on the DM mass.

Our observations generalize to other scotogenic models such as those with triplet fermions [33] and/or singlet-doublet scalars [34], where the neutrino mass matrices take forms similar to Eq. (8). However, since the neutral components of electroweak triplets can annihilate into gauge bosons, the Yukawas and LFV processes are generally smaller, so that collider constraints must also be considered $[35,36]$.

\section{ACKNOWLEDGMENTS}

The authors thank C. Weinheimer for very helpful discussions and comments on the manuscript. This work has been supported by the DFG through the Research Training Network 2149 "Strong and weak interactionsfrom hadrons to dark matter".
[1] M. Tanabashi et al. (Particle Data Group), Phys. Rev. D 98, 030001 (2018).

[2] M. Klasen, M. Pohl, and G. Sigl, Prog. Part. Nucl. Phys. 85, 1 (2015).

[3] E. Ma, Phys. Rev. D 73, 077301 (2006).

[4] D. Restrepo, O. Zapata, and C. E. Yaguna, J. High Energy Phys. 11 (2013) 011.

[5] Y. Cai, J. Herrero-García, M. A. Schmidt, A. Vicente, and R. R. Volkas, Front. Phys. 5, 63 (2017).

[6] Y. Fukuda et al. (Super-Kamiokande Collaboration), Phys. Rev. Lett. 81, 1562 (1998).

[7] Q. Ahmad et al. (SNO Collaboration), Phys. Rev. Lett. 87, 071301 (2001).

[8] Q. Ahmad et al. (SNO Collaboration), Phys. Rev. Lett. 89, 011301 (2002).

[9] M. Aker et al. (KATRIN Collaboration), Phys. Rev. Lett. 123, 221802 (2019).

[10] G. Drexlin, V. Hannen, S. Mertens, and C. Weinheimer, Adv. High Energy Phys. 2013, 293986 (2013).

[11] S. Vagnozzi, E. Giusarma, O. Mena, K. Freese, M. Gerbino, S. Ho, and M. Lattanzi, Phys. Rev. D 96, 123503 (2017).

[12] N. Aghanim et al. (Planck Collaboration), arXiv:1807 .06209 .

[13] I. Esteban, M. Gonzalez-Garcia, A. Hernandez-Cabezudo, M. Maltoni, and T. Schwetz, J. High Energy Phys. 01 (2019) 106.

[14] J. Casas and A. Ibarra, Nucl. Phys. B618, 171 (2001).

[15] T. Toma and A. Vicente, J. High Energy Phys. 01 (2014) 160.

[16] A. Vicente and C. E. Yaguna, J. High Energy Phys. 02 (2015) 144.

[17] G. Aad et al. (ATLAS and CMS Collaborations), Phys. Rev. Lett. 114, 191803 (2015).

[18] J. Kubo, E. Ma, and D. Suematsu, Phys. Lett. B 642, 18 (2006).
[19] G. Abbiendi et al. (OPAL Collaboration), Phys. Lett. B 572, 8 (2003).

[20] M. Lindner, M. Platscher, C. E. Yaguna, and A. Merle, Phys. Rev. D 94, 115027 (2016).

[21] A. Baldini et al. (MEG Collaboration), Eur. Phys. J. C 76, 434 (2016).

[22] F. Renga (MEG Collaboration), Hyperfine Interact. 239, 58 (2018).

[23] U. Bellgardt et al. (SINDRUM Collaboration), Nucl. Phys. B299, 1 (1988).

[24] A. Blondel et al., arXiv:1301.6113.

[25] C. Dohmen et al. (SINDRUM II Collaboration), Phys. Lett. B 317, 631 (1993).

[26] The PRISM/PRIME Group, LOI on experiments at the J-PARC $50 \mathrm{GeV}$ PS, http://j-parc.jp/researcher/Hadron/en/ pac_0606/pdf/p20-Kuno.pdf.

[27] W. Porod and F. Staub, Comput. Phys. Commun. 183, 2458 (2012).

[28] G. Belanger, F. Boudjema, A. Goudelis, A. Pukhov, and B. Zaldivar, Comput. Phys. Commun. 231, 173 (2018).

[29] J. Harz, B. Herrmann, M. Klasen, K. Kovarik, and P. Steppeler, Phys. Rev. D 93, 114023 (2016).

[30] J. Branahl, J. Harz, B. Herrmann, M. Klasen, K. Kovarik, and S. Schmiemann, Phys. Rev. D 100, 115003 (2019).

[31] M. Klasen, C. E. Yaguna, J. D. Ruiz-Alvarez, D. Restrepo, and O. Zapata, J. Cosmol. Astropart. Phys. 04 (2013) 044.

[32] A. Ibarra, C. E. Yaguna, and O. Zapata, Phys. Rev. D 93, 035012 (2016).

[33] E. Ma and D. Suematsu, Mod. Phys. Lett. A 24, 583 (2009).

[34] Y. Farzan, Phys. Rev. D 80, 073009 (2009).

[35] M. Cirelli, F. Sala, and M. Taoso, J. High Energy Phys. 10 (2014) 033; 01 (2015) 041(E).

[36] P. B. Dev and R. Mohapatra, Phys. Rev. Lett. 115, 181803 (2015). 